\title{
ORIGINAL
}

\section{EL DISCURSO DE LOS PROFESIONALES DE ATENCIÓN PRIMARIA DE LA COMUNIDAD DE MADRID SOBRE EL TRABAJO CON GRUPOS: SENTIDO, FINALIDADES Y ÁMBITOS DE INTERVENCIÓN (*)}

\section{Juan Carlos Duro Martínez (1,2)}

(1) Centro Universitario de Salud Pública. Comunidad de Madrid-Universidad Autónoma de Madrid.

(2) Departamento de Medicina Preventiva, Salud Pública e Historia de la Ciencia. Universidad Complutense de Madrid.

(*) Este artículo es parte de una investigación financiada por el Fondo de Investigaciones Sanitarias (Exp. 93/0907) en la que el autor fue el investigador principal y en la que también participó como investigadora la psicóloga Celia Arroyo.

\section{RESUMEN}

Fundamento: La Educación para la salud con grupos es una actividad cada vez más importante en atención primaria. Con el objetivo de contribuir a su desarrollo, esta investigación se plantea conocer los discursos de los profesionales sanitarios sobre las prácticas grupales y específicamente sobre su sentido, finalidades y ámbitos de aplicación.

Métodos: Desde una perspectiva cualitativa-estructural se realizaron cuatro grupos de discusión y cuatro entrevistas abiertas con médicos generales, especialistas en Pediatría, en Medicina Familiar y Comunitaria, Enfermería y Trabajo Social, de las Áreas de Atención Primaria de la Comunidad de Madrid.

Resultados: Los discursos de los profesionales de atención primaria sobre los grupos se inscriben dentro de las actividades de educación para la salud, siendo ésta una actividad supeditada a la consulta individual. Para hacer grupos los profesionales incluyen argumentos del orden de la jerarquía, eficiencia, eficacia y satisfacción personal. Los principales colectivos objeto del trabajo en grupo son, por este orden, los pacientes crónicos (hipertensos y diabéticos), mujeres (perimenopaúsicas, con malestar general, etc.), escolares y profesores, asociaciones de la comunidad y cuidadores de enfermos crónicos.

Conclusiones: Los profesionales de atención primaria mayoritariamente consideran el trabajo con grupos como algo marginal cuya finalidad principal sería el ahorro y, subsidiariamente, la Educación para la salud. Se realizan básicamente con pacientes crónicos y mujeres, planteándose el trabajo comunitario en un segundo término.

Palabras clave: Educación para la salud. Atención primaria de salud. Investigación cualitativa. Grupo de discusión. Entrevista abierta.

\section{Correspondencia:}

Juan Carlos Duro Martínez

Centro Universitario de Salud Pública.

C/ General Oráa, 39

28006 Madrid Tfno: 914117572 Fax: 914116696

Correo electrónico: juancarlos.duro@uam.es

\section{ABSTRACT}

\section{The Opinions of the Primary Care Professionals of the Community of Madrid Concerning the Work with Groups: Reason for Being, Purposes and Fields of Intervention}

Background: Health Education with groups is an increasingly important activity in primary care. For the purpose of contributing to the furthering thereof, this research is aimed at ascertaining the opinions of the health care professionals with regard to the group practices and particularly with regard to their reason for being, purposes and fields of intervention.

Methods: From a qualitative-structural perspective, four discussion groups and four open interviews were held with general practitioners, specialists in Pediatrics, in Family and Community Medicine, Nursing and Social Work in the Primary Care Areas in the Autonomous Community of Madrid.

Results: The opinions of the primary care professionals regarding the groups are confined to the activities of Health Education, this being an activity subject to individual inquiry. To form groups, the professionals include arguments on the order of hierarchy, efficiency, effectiveness and personal satisfaction. The main groups regarding which the group work was done are chronic patients (hypertension and diabetes), women (pre-menopause, general malaise), school-age children and teachers, community associations and caregivers of the chronically ill.

Conclusions: The majority of the primary care professionals consider the work with groups to be something on the side, the end purpose of which would be savings and, on a secondary scale, Health Education. These groups mainly regard chronic patients and women, community work being approached on a second plane.

Keywords: Health Education. Primary health care. Qualitative research. Discussion group. Open interview. 


\section{INTRODUCCIÓN}

Los discursos y las prácticas grupales en la atención primaria de salud en nuestro país durante los últimos veinticinco años han ido evolucionando desde las pioneras experiencias comunitarias que contribuyeron a la Reforma sanitaria ${ }^{1-4}$ hasta su implantación a lo largo de los 90, con la indudable influencia de las recomendaciones del Informe Abril $^{5}$ y la puesta en marcha de la Cartera de Servicios $^{6}$. En todo este recorrido el trabajo con grupos ha estado presente, ya sea en sus planteamientos más cercanos a la promoción de la salud y la participación comunitaria $^{7,8}$, ya en los acercamientos más tecnológicos vinculados a la asistencia y a la Educación para la Salud ${ }^{9,10}$.

Las resistencias al trabajo en y con grupos atraviesan a las instituciones y a los propios profesionales. Se convierte así en primordial conocer la perspectiva de los profesionales sanitarios sobre el trabajo psicosocial más relevante, los grupos, como una manera de conocer sus actitudes, valores, representaciones, etc., sobre un tipo de prácticas que, con frecuencia, se potencian en lo discursivo pero que escasean en la práctica cotidiana.

Conocer el discurso sobre las prácticas grupales en lo referente a la promoción y educación para la salud en atención primaria cuando menos puede servir para la formación de estos profesionales en orden a potenciar un modelo psicosocial e interdisciplinar de intervención, así como para la adopción de medidas de carácter organizativo y de cambio institucional que posibiliten dichas prácticas.

En el marco de ese metaobjetivo llevamos a cabo esta investigación en la que hemos utilizado, en el nivel tecnológico, como dispositivo fundamental de captura de datos - discursos - el grupo de discusión, a decir de Jesús Ibáñez ${ }^{11-13}$, principal instrumento técnico dentro de la perspectiva estructural para la investigación en ciencias sociales ${ }^{11,14}$.

El objetivo general de esta investigación es conocer los discursos de los profesionales sanitarios de atención primaria sobre las prácticas grupales en promoción y educación para la salud. Los objetivos específicos, de los que da cuenta este artículo, son: identificar los significados y sentidos que los distintos profesionales dan a dicho trabajo en su cotidianidad y conocer sus motivaciones y los distintos ámbitos de intervención con grupos en atención primaria.

\section{SUJETOS Y MÉTODO}

A tenor del carácter y naturaleza de los objetivos de investigación propuestos, que pertenecen al segundo nivel de la realidad social —el orden del discurso- - , se eligió el método estructural-cualitativo como el más adecuado para darles respuesta. Este método de investigación toma como centro a los sujetos-actores (en nuestro caso, los profesionales sanitarios de los Equipos de Atención Primaria del Insalud de la Comunidad de Madrid) para tratar de captar la riqueza de las experiencias de las personas en sus propios términos, palabras y visiones, intentando entender el mundo subjetivo de los participantes del estudio ${ }^{15}$. Como técnicas de captura de datos se utilizó el grupo de discusión y como complemento las entrevistas abiertas ${ }^{16,17}$

\section{Universo de estudio y clases estructurales}

La población objeto de nuestro estudio - universo- está constituida por médicos/as y enfermeros/as, y por los trabajadores/as sociales que se han incluido en los Equipos de Atención Primaria. Excluimos del estudio a otros integrantes de los equipos, bien por no tener funciones y responsabilidades directas de atención a la salud de la población — auxiliares administrativos- 
bien por su escasa relevancia cuantitativa o cualitativa en la misma - fisioterapeuta, auxiliares de clínica, etcétera.

Los discursos de los profesionales de atención primaria sobre las prácticas grupales no pueden ser homogéneos dadas las diferentes «clases estructurales» relativas al tema de estudio. Podemos por lo tanto dividir a nuestra población objeto de estudio en función de diferentes variables relevantes para nuestros objetivos de investigación: profesión, área sanitaria y género. El resto de variables hay que tenerlas en cuenta para buscar la mayor heterogeneidad en el discurso de cada clase. Son especialmente relevantes: el tiempo de actividad laboral y, sobre todo, si han tenido formación y/o experiencia de trabajo grupal y/o psicosocial.

\section{Muestra y diseño de los Grupos de Discusión}

Dado que en un estudio cualitativo la significación de la muestra viene dada por su representatividad estructural (tablas 1 y 2), y teniendo en cuenta los criterios de homogeneidad para la producción del discurso grupal de manera que no se produzcan blo- queos en la comunicación y se dificulte la producción grupal, se organizaron los grupos en función de la variable profesión que tomamos como heterogeneidad excluyente y dentro de la profesión de médicos, se discriminó entre médicos generales, sin especialidad, médicos especialistas en pediatría y médicos especialistas en medicina familiar y comunitaria (heterogeneidad incluyente). La variable Área sanitaria se tuvo en cuenta para evitar la excesiva representación de aquellas áreas en las que hubiera un mayor desarrollo de las actividades grupales por diversas razones (mayor formación y experiencias de los profesionales, más potenciación por parte de las gerencias, etc.). La variable experiencia previa con el trabajo con grupos, aunque fue tenida en cuenta a la hora de la composición de los grupos, no ha podido ser identificada cuantitativamente en cada grupo por no haber llevado los investigadores un registro de la misma (es decir que en cada grupo había profesionales con distintos grados de experiencia pero no hemos cuantificado cuántos tenían experiencia y en qué grado) ya que fueron los contactadores los que la tuvieron en cuenta a la hora de seleccionar a los participantes en los grupos de discusión. Esta limitación pensamos que no quita validez a los resultados en tanto

Tabla 1

Diseño de los Grupos de discusión y las entrevistas abiertas

\begin{tabular}{|c|c|c|c|c|}
\hline & Profesionales & Área Sanitaria & Titulación & Sexo/Edad \\
\hline $\begin{array}{l}\text { Grupo de } \\
\text { Discusión } 1\end{array}$ & $\begin{array}{l}9 \text { profesionales de } \\
\text { enfermería }\end{array}$ & $\begin{array}{c}\text { Áreas } 1,2,5,9, \\
10 \text { y } 11\end{array}$ & & $\begin{array}{c}8 \text { mujeres y } 1 \text { hombre } \\
30-40 \text { años }\end{array}$ \\
\hline $\begin{array}{l}\text { Grupo de } \\
\text { Discusión } 2\end{array}$ & 7 médicos/as & $\begin{array}{c}\text { Áreas } 1,3,6,8, \\
10 \text { y } 11\end{array}$ & $\begin{array}{c}\text { Medicina de Familia } \\
\text { y Pediatría }\end{array}$ & $\begin{array}{c}4 \text { mujeres y } 3 \text { hombres } \\
30-45 \text { años }\end{array}$ \\
\hline $\begin{array}{l}\text { Grupo de } \\
\text { Discusión } 3\end{array}$ & 5 trabajadoras sociales & $\begin{array}{c}\text { Áreas } 1,8,9 \\
\text { y } 10\end{array}$ & & $\begin{array}{l}5 \text { mujeres } \\
26-35 \text { años }\end{array}$ \\
\hline $\begin{array}{l}\text { Grupo de } \\
\text { Discusión } 4\end{array}$ & 6 médicos & $\begin{array}{c}\text { Áreas } 4,7,8,9 \\
\text { y } 11\end{array}$ & $\begin{array}{l}\text { Medicina General } \\
\text { y de Familia }\end{array}$ & $\begin{array}{c}5 \text { mujeres y } 1 \text { hombre } \\
30-40 \text { años }\end{array}$ \\
\hline $\begin{array}{l}2 \text { Entrevistas } \\
\text { en profundidad }\end{array}$ & médicos & Áreas 3 y 6 & Medicina General & $\begin{array}{l}\text { hombres } \\
35-40 \text { años }\end{array}$ \\
\hline $\begin{array}{l}2 \text { Entrevistas } \\
\text { en profundidad }\end{array}$ & trabajadoras sociales & Áreas 2 y 5 & & $\begin{array}{l}\text { mujeres } \\
30 \text { años }\end{array}$ \\
\hline
\end{tabular}


Tabla 2

Variables para la composición de los Grupos de Discusión

- Colectivo profesional de pertenencia: médicos generales y médicos especialistas en pediatría, médicos especialistas en medicina familiar y comunitaria, enfermeros y trabajadores sociales.

- Área Sanitaria en la que se desempeña el trabajo

- Hombres y mujeres

- Un mínimo de dos años de experiencia en Atención Primaria

- Formación/experiencia en trabajo con grupos.

éstos expresan la tendencia del posicionamiento topológico de las opiniones en función de los distintos niveles de experiencia.

El proceso de captación empleado ha sido el método de «bola de nieve» o «red de captación mediante pasos sucesivos», utilizándose en un primer paso captadores que por motivos de amistad, de relación profesional, vínculos a través de la actividad docente o varios de ellos simultáneamente, tuvieran una buena relación con los investigadores.

\section{Realización de los Grupos de Discusión y las Entrevistas Abiertas}

Los cuatro grupos de discusión y las cuatro entrevistas abiertas que forman parte de esta investigación se realizaron en el Centro Universitario de Salud Pública. La duración de cada reunión de grupo osciló entre los setenta y cinco y los noventa minutos, dándose por finalizado el grupo cuando, a juicio del preceptor, se había agotado la producción grupal sobre el tema y empezaba a producirse ostensible redundancia sobre el mismo. En todos los grupos y entrevistas se invitó a la discusión mediante la consigna o intervención inicial indirecta, pues no explicitaba el objetivo de la investigación: vamos a hablar del trabajo cotidiano que se hace fuera de la consulta en los equipos, para después, según la guía temática (tabla 3 ), ir centrando el debate en las actividades o trabajos con grupos, una vez que se nombraba de forma espontánea por los hablantes.
Tabla 3

Guía temática para los grupos de discusión y entrevistas referida a los objetivos específicos que se presentan en este artículo

- Actividades no asistenciales que se realizan en Atención Primaria de Salud en Madrid.

- Valoración sobre actividades grupales y con la comunidad hechas por los profesionales de APS.

- Razones por las que se hacen grupos en atención primaria.

- Ventajas e inconvenientes de las actividades grupales con pacientes en el Centro de Salud y con la comunidad.

- Ámbitos de intervención grupal en atención primaria.

Todo lo dicho en los grupos de discusión y entrevistas abiertas fue recogido en cinta magnetofónica y posteriormente transcrito de forma literal. Ello constituye el corpus de datos discursivos de esta investigación, y sobre él se aplicó el análisis e interpretación, que se refleja en los resultados.

El análisis y la interpretación de la información recogida se efectuó, en primera instancia, por el investigador/preceptor que realizó los grupos de discusión y posteriormente fue contrastado y supervisado por el investigador principal del estudio. Ninguno de los dos investigadores conocía ni tuvo contacto previo con los integrantes de los grupos ni con los participantes en las entrevistas abiertas. Acorde con ese planteamiento teórico-metodológico y puesto que no se trataba de una investigación-acción ${ }^{18}$ no se «devolvió» el resultado a los informantes directos, si bien como «informe preliminar» se utilizó en actividades formativas con profesionales de atención primaria (Área 2 del Insalud, Diplomado de Sanidad de la Comunidad de Madrid, etc.) contrastando los resultados con la experiencia y opiniones de los profesionales de atención primaria que participaban en estos cursos.

\section{RESULTADOS}

El análisis de los resultados se hizo según la guía de los objetivos planteados. Su organización en epígrafes facilita la exposición de los resultados que se acompañan de los dichos (verbatim) más relevantes (tabla 4). 
Tabla 4

«Dichos» (verbatim) más relevantes

N. ${ }^{\circ} 1$ «Mira, si nos pusiésemos a hacer cálculos de lo que le podríamos ahorrar al Estado haciendo una educación para la salud buena, es decir, por ejemplo el tabaco, yo conozco el tabaco, yo con que dejen 20 personas al año, si eso lo sumamos en los días que supuestamente tenía que estar pues que la gente que fume, que tiene un cáncer y todo eso, lo cuantificas, y son muchos millones de pesetas, yo creo que es superinteresante...» Grupo de Discusión de Enfermería (GDE).

N. ${ }^{\circ} 2$ «... Los objetivos que se plantean son esos, primero descargar las consultas, evidentemente no todo el que pasa a la consulta necesita receta, ¿entiendes?» Grupo de Discusión de Trabajadoras Sociales (GDTS)

$\mathrm{N}^{\circ}{ }^{\circ} 3$ «Es lo que... o sea partimos de que la educación grupal va luego a revertir en que vamos a tener menos carga... porque va a estar mejor educada la población y entonces no va a demandar tanto, porque vas a educar en vez de a uno a diez a la vez o a doce... y además se lo van a aprender» (GDE).

N. ${ }^{\circ} 4$ «Y juntar a un grupo de veinte o quince o treinta padres, o cinco, que te van a llevar quizá el mismo tiempo, veinte minutos, $y$ les expliques lo que a todos igual les explicas... igual hasta salen más reforzados. En ese cuarto de hora que yo tengo de consulta programada, que me voy a llevar a un solo niño, en ese cuarto de hora igual me puedo llevar a diez o doce padres...» Grupo de Discusión de Médicos (GDM).

N. 5 «Bueno, no sé si exactamente se recibió mal... no sé cómo decirlo... Se vivió como algo que hay que sacar adelante, que no había otra, o te montas en este carro o a ver qué pasa aquí, porque tenemos que rentabilizar, ¿entiendes?, y eso a mi personalmente me encantó porque estaba en mi... pero a lo mejor a otras compañeras...» (GDTS)

N. ${ }^{\circ} 6$ «A mi si me gusta el tema de grupos, me parece necesario... y entonces se ha puesto en marcha... cosa que a mi me tranquilizó, porque dije, bueno, ya no es sólo misión nuestra sino que hay algo que se está poniendo en marcha...»(GDM)

N. ${ }^{\circ} 7$ «Eso está clarísimo, que educación para la salud hay que hacerla (...). O sea, que esta educación hay que hacerla, y que tanto grupal como individual» (GDM)

N. ${ }^{\circ}$ «... por lo menos se intenta el cambiar los hábitos alimentarios de los niños... te estoy hablando de un colegio al lado de nuestro centro de salud con el que estuvimos trabajando tres años... entonces ahí se intentó cambiar estos hábitos... por ejemplo los desayunos...» (GDM).

N. ${ }^{\circ} 9$ «El trabajo con el grupo es preventivo, y te da básicamente a conocer la enfermedad o el problema, y ayudar a autocontrolarse, a autocurarse digamos. Ésa es la ventaja del grupo, yo creo que preventivo y educacional... digamos que la prevención y la educación es muy rentable, muy rentable terapéuticamente hablando, ¿entiendes?, a nivel de control de la enfermedad, el autocontrol en esa enfermedad es estupendo, y basta con educarlos» Entrevista con Médico General (EMG).

N. ${ }^{\circ} 10$ «Lo que pasa que el objetivo básico tiene que ser siempre el mismo, que la mujer, iplas!, dé el salto, promoción, trabajo, o seguir estudiando, me da igual, pero que haya ese salto, ese tiene que ser siempre el objetivo, ¿no?, yo creo» Entrevista con Trabajadora Social (ETS).

N. ${ }^{\circ} 11$ «Estamos muy empeñados en primaria, o en lo que yo vivo, en diabetes, hipertensión, colesterol, pues a la gente es que la tengo con la hipertensión, con la diabetes y con la salud escolar.» (GDTS).

$\mathrm{N} .{ }^{\circ} 12$ «Yo creo que ahi hemos exagerado, nos hemos pasado y luego no nos queda tiempo muchas veces para ver a otros pacientes. Yo... me parece que por ejemplo la enfermería dedica demasiado tiempo a hipertensos, a diabéticos, a toda esta gente, los está viendo en demasía, es que ya es una pérdida de tiempo, yo lo veo como pérdida de tiempo» (GDM).

N. ${ }^{\circ} 13$ «Entonces yo ahora digo que puedo llevar un grupo sola pero no lo llevo porque no tengo una suficiente demanda, no planteo ni de diabéticos ni de hipertensos, yo hablo de frecuentadores de consulta, mujeres que se han divorciado, mujeres que su marido es alcohólico, mujeres que su hijo es minusválido, mujer que es viuda; yo no hablo de hipertensos ni de diabéticos. Pero no hay ni demanda» (GDTS)

N. ${ }^{\circ} 14$ «Entonces, hay una asociación de mujeres que nos reclama para dar menopausia, pues va un médico y una enfermera, hay una asociación que te reclama, o los colegios te reclaman, queremos charlas de tal y tal y se lo apuntamos dentro de nuestras posibilidades» (GDM)

N. ${ }^{\circ} 15$ «...este año hemos presentado los servicios del centro de salud en nuestros nueve colegios de referencia presentando el Programa de Atención al Niño, y de ahi pues ya nos han llovido bastantes peticiones para EPS...» (GDTS).

N. ${ }^{\circ} 16$ «... hay algo que se está poniendo en marcha que se llama Plan Comunitario, que lo lleva la asociación de... bueno, es una manera de estructurar todos los servicios que hay en el distrito (...), a mi lo que me gusta de esto es que no es una institución más sino que es la coordinación entre todas...» (GDM)

N. ${ }^{0} 17$ «Hay una parte que me gustaría comentar, que es la parte comunitaria, que a todo el mundo le resulta más dificil, la educación para la salud, porque normalmente todo eso de participación comunitaria viene a ser educación para la salud, formar grupos o salir fuera del centro para hacer charlas con diferentes colectivos... no es participación comunitaria de, bueno, que los miembros de la comunidad participen en lo que es el desarrollo del centro, sino en esa otra vertiente de participación que es a través de la educación para la salud...» (GDTS).

N. ${ }^{\circ} 18$ «Lo que os contaba de los cuidadores de personas incapacitadas... eso si no lo hacemos nosotros no se encarga ninguna institución, y necesitan mucha ayuda, tienen que saber muchas cosas» (GDM). 
Los grupos en Atención Primaria: «lo que se hace si queda tiempo"

El trabajo con grupos aparece junto con otras actividades que también se hacen «fuera de la consulta» como investigación, formación, sesiones clínicas, reuniones de equipo, etc. Todos estos temas se ponen en relación metonímica con opiniones sobre la organización interna de los equipos, las relaciones entre los equipos y las Gerencias, la pertinencia de determinados registros (muchos de ellos se perciben como «burocracia sin sentido»), la adecuación de ciertos programas, las relaciones entre colectivos profesionales, etc. En suma, el trabajo con grupos suscita reflexiones en relación con el sentido que el trabajo cotidiano en atención primaria tiene para sus profesionales en distintos ámbitos: en cuanto a la tarea, eficacia, etc.; en cuanto a la identidad profesional, reconocimiento, expectativas, etc.; en cuanto a la esfera afectivo-relacional, en lo que hace al clima de los equipos, etcétera.

En concreto, las referencias más explícitas al trabajo con grupos surgen casi siempre alrededor de la educación para la salud (en sentido amplio y con distintos modelos teóricos en uso) y suele ser un discurso que pone de manifiesto un conflicto dinámico y lejos del consenso. Tanto el conflicto como el disenso aparecen al hablar del propio sentido del trabajo con grupos, su eficacia, los objetivos que persigue, la disponibilidad para realizarlo, las motivaciones y grado de implicación de los distintos profesionales, etc. Además, el polo de comparación del trabajo con grupos es siempre la atención o asistencia individual, constituida en eje central de la cotidianeidad de los equipos. El trabajo con grupos aparece — con distintos grados y según quiénes sean los hablantescomo «lo otro» (dentro del contexto de la educación para la salud versus la tarea asistencial-individual), «lo que se hace si queda tiempo», una actividad de segunda, lo deseable, etc.

\section{¿Por qué y para qué se hacen los grupos?}

En el discurso de los profesionales sobre las razones explicitadas para justificar la realización de grupos en atención primaria se entremezclan argumentos en relación con el pensamiento/valoración socio-profesional de los grupos; y relacionadas más propiamente con los objetivos o beneficios directos que se pretenden alcanzar con el trabajo con grupos.

\section{Rentabilidad económica: «... los grupos es ahorro y no me digas que no"}

El carácter rentable y de ahorro (en tiempo, esfuerzo, dinero y recursos humanos) del trabajo con grupos parece ser una de las teorías aceptadas con más fuerza entre los profesionales sanitarios de atención primaria. Constituye casi un a priori para justificar desde fuera la realización de grupos en distintos ámbitos, al margen de consideraciones de otros órdenes. De alguna forma, la necesidad de ahorrar - y la posibilidad de hacerlo, entre otros medios, a través de los grupos - parece haber sido asumida o hecha suya por algunos profesionales como un objetivo "razonable», perteneciente en primera instancia a la Administración. Este «hacer suyo» este argumento pasaría por percibir los beneficios del ahorro en el trabajo cotidiano de cada profesional.

Si los objetivos educativo-terapéuticos del trabajo con grupos (como una forma más de educación sanitaria o para la salud) se cumplen, el beneficio secundario - pero directo- sería la disminución de enfermedades y el ahorro consiguiente en el presupuesto global del sistema sanitario (Verbatim 1).

\section{Descarga de las consultas y ahorro de tiempo}

La descarga de las consultas es el efecto más deseable por parte del colectivo médi- 
co, apuntado también como positivo por parte de los otros colectivos. La presión asistencial disminuiría porque se tratarían fuera de la consulta problemas que ahora se tratan dentro, porque la población aprendería a no demandar tanto, porque, incluso, se daría respuesta más «adecuada» a demandas ahora no satisfechas: como reflejan las citas siguientes, estas demandas irían en la línea de «compartir el problema con otra gente», "educar a la población», o "algo distinto a la receta», aspectos que se incluyen como esenciales del trabajo con grupos y no de la consulta individual (Verbatim 2 y 3 ).

El ahorro en tiempo también está relacionado con la descarga de la consulta, ya que atender simultáneamente a un número de personas con demandas parecidas lleva menos tiempo que hacerlo individualmente. El significado implícito en esta definición apunta a una concepción del grupo como un «auditorio» (muchos espectadores que escuchan algo) y del tiempo como el que el profesional tarda en «explicar» algo a dicho auditorio (Verbatim 4).

\section{Es obligatorio: "...ahora hay que hacerlo porque está en Cartera de Servicios"}

La inclusión de las actividades de grupos en la Cartera de Servicios se interpreta, entre otras cosas, como una consecuencia de la estrategia de reducción de costes y, menos, como estrategia de mejora de la calidad. Desde el momento en el que constituye una actividad de la que hay que dar cuenta en las memorias anuales, se convierte en algo obligatorio: se quiera o no, es una razón para llevar a cabo trabajo con grupos (Verbatim 5).

Entre los profesionales sanitarios hay, al menos, dos posturas ante la inclusión de los grupos en la Cartera de Servicios. Aquéllos menos convencidos del valor y eficacia del trabajo con grupos lo observan como una imposición, como una actividad más a sumar a las que ya están establecidas. Éstos apuntan a que el trabajo con grupos sigue siendo algo secundario, incluso aunque el documento lo refleje y obligue a su realización. Sin embargo, los profesionales con una actitud más favorable a la actividad reciben positivamente su inclusión en la Cartera de Servicios a partir de la percepción de un doble beneficio: por un lado, aporta un soporte formal por parte de la Administración en el que enmarcar actividades o tareas que se venían haciendo con carácter voluntario o, incluso, voluntarista; por otro, desde el convencimiento del valor de los grupos en el trabajo cotidiano, observan la «imposición» como un punto de partida, desde el que otros profesionales pueden participar. En otras palabras, estos profesionales se ven apoyados desde lo «instituido», disminuyendo la sensación de ser lo «instituyente» (con la connotación de oposición que siempre tiene esto último) (Verbatim 6). En todo caso, parece haber una postura mayoritaria, más bien pasiva, que tomaría como centro la consideración de la realización de grupos como una actividad "extra», fuera de las obligaciones establecidas y, en general, como algo que se hace siempre en segundo término y de manera voluntarista.

\section{Sirven para prevenir y fomentar los autocuidados}

Los profesionales más afines a esta forma de trabajar establecen que los objetivos que se persiguen con el trabajo con grupos son, en su esencia, los de la educación para la salud individual (Verbatim 7). Dichos objetivos están fundamentalmente relacionados con la prevención; así, eliminar hábitos o conductas «insanas», implantar hábitos saludables, provocar una toma de conciencia sobre los estilos de vida inconvenientes o perjudiciales (siempre en el sentido de hábitos o conductas), etc. (Verbatim 8). En el caso de personas con enfermedades crónicas se busca aumentar su información sobre dichas enfermedades. En la misma línea, se persigue promocionar los autocuidados de los pacientes que requieren atención continuada. Con ello se busca una mayor autonomía de la población con respecto al sistema 
sanitario que, indirectamente, supondría un ahorro si consiguiera disminuir la demanda asistencial. En este sentido, se está hablando del grupo como un dispositivo «terapéutico», persiguiéndose el autocontrol de la enfermedad y la menor dependencia del médico por parte del enfermo (Verbatim 9).

Además de estos objetivos de orden clínico-terapéutico, los colectivos de enfermería y trabajadores sociales apuntan también otros objetivos deseables de orden psicosocial o, en términos más amplios, de promoción de la salud. Al hablar de estos objetivos, estos colectivos profesionales informan también de los grupos de población a los que se refieren, en general, población «sana» desde el punto de vista clínico, pero quizá «enferma» desde el punto de vista psicosocial (sobre todo, los usuarios de la consulta de trabajo social) (Verbatim 10). El trabajo con grupos favorecería la creación de espacios de escucha de problemas que no tienen cabida en otros lugares del trabajo cotidiano de la atención primaria, supondrían el inicio de grupos de autoayuda de personas con necesidades parecidas y sería un punto de partida para procesos de «maduración personal». Tanto el colectivo de enfermería como el de trabajadores sociales consideran que el tercer colectivo, el médico, no participa, en general, de estos objetivos, por lo que la posibilidad de llevar a la práctica grupos bajo este enfoque no suele contar con su apoyo.

\section{Ámbitos de trabajo con grupos}

Los ámbitos de trabajo con grupos en atención primaria, en orden descendente de importancia, son: personas con patologías crónicas, mujeres, salud escolar, participación comunitaria y formación de cuidadores.

\section{Patologías crónicas: "... aquí es un chollo ser hipertenso"}

El primer ámbito espontáneo con el que se asocia el trabajo con grupos es el de los pacientes con patologías crónicas, en especial la hipertensión y la diabetes. Siguiendo el discurso de los profesionales, éste parece ser uno de los ámbitos más estructurados y sistematizados a través de los protocolos, en los que el trabajo con grupos empieza a tener un lugar más o menos establecido y fijo. El interés por este ámbito coincide con el interés más general (materializado en protocolos estructurados) de la atención primaria por las patologías crónicas en la consulta individual y responde a una de las actividades protocolizadas en la Cartera de Servicios (Verbatim 11). Además, la propia Cartera de Servicios refleja esta «sistematización» de las actuaciones a llevar a cabo con los pacientes que sufren este tipo de patologías, fijando, a través de las Normas Técnicas Mínimas, un conjunto de actividades planificadas según un protocolo y realizando evaluaciones pre-test y post-test de conocimientos, habilidades y actitudes de los participantes en los grupos.

En este sentido, se dice que desde que a un usuario se le diagnostica hipertensión o diabetes entra inmediatamente en el círculo de actividades y actuaciones previstas para esta circunstancia, entre las que se incluyen «sesiones de grupo» para familiarizarse y tomar contacto con su enfermedad.

Aún reconociendo que estas patologías son frecuentes y aparecen realmente en las consultas constituyendo una parte importante de la demanda asistencial, entre algunos profesionales surge una crítica de cierta «arbitrariedad» en la selección de estas dos patologías como objeto de interés y atención. La decisión de dedicarles tiempo, recursos humanos y planificación, supone el dejar de lado a otros pacientes con patologías distintas que se verían perjudicados porque no existe protocolo para ellos y que podrían beneficiarse de una mejor atención. Así, la expresión de que «aquí es un chollo ser hipertenso» pone de manifiesto que algunos profesionales más críticos (de los tres colectivos, pero de forma más explícita en trabajo social) perciben una cierta desigual- 
dad en el reparto de dicha atención (o ruptura de la equidad) y, complementariamente, que si uno «tiene la suerte» de sufrir hipertensión (u otra patología «protocolizada») se convierte en beneficiario de más servicios por parte del Centro de Salud (controles, seguimientos, grupos, etc.) que otros pacientes (Verbatim 12). La queja desde los trabajadores sociales se enmarca, también, en una demanda doble: de aprovechamiento de su propia formación, no estrictamente sanitaria sino psicosocial o sociosanitaria en sentido amplio, y de un lugar reconocido en el seno de los equipos donde su actuación tenga sentido. Esta posición del colectivo de trabajo social autopercibida como "periférica» (y difusa) explica y anima gran parte de las reflexiones sobre el trabajo con grupos desde su punto de vista (Verbatim 13).

Interpretando las posiciones detectadas en el discurso, vemos que la decisión de dedicar más atención a ciertas patologías (diabetes, hipertensión, etc.) no tiene, en realidad, un carácter arbitrario, sino que responde al Modelo Médico Hegemónico, con sus «intereses» y su saber (centrado en aspectos clínico-curativos e individuales, incluso aunque se trabajen en grupo: como suma de individuos). La postura proveniente de trabajo social (por su formación y su posición dentro de la organización) apunta más a la reivindicación del modelo psicosocial, incorporando, como aspectos a considerar, determinantes de la salud no sólo clínicos sino también sociales, y observando el grupo en su carácter interactivo-relacional y como dispositivo de socialización y aprendizaje. Los discursos de los profesionales de atención primaria respecto a sus actitudes, relaciones entre los profesionales y modalidades y técnicas grupales, son otros objetivos específicos de esta investigación que serán objeto de una posterior publicación.

\section{Grupos con mujeres}

El trabajo con grupos de mujeres perimenopáusicas, a las que se define de forma dominante por una situación vital «medicalizada» (teniendo en cuenta los cambios hormonales y sus consecuencias clínicas en esa época de la vida de la mujer), se hacen tanto a demanda como de manera programada, en el sentido de que la iniciativa surja de algún pr2ofesional del equipo (sobre todo, de enfermería y trabajo social). Con la expresión «a demanda» los profesionales se suelen referir a peticiones al Centro de Salud de charlas o colaboración de algunos grupos de mujeres ya establecidos en la comunidad (a través de Asociaciones o similar). En estos casos, se organiza, en función de las posibilidades, un programa que dé respuesta a las peticiones y se reparte la realización entre los profesionales más «motivados». En todos los grupos de discusión se puso de manifiesto que la realización o no de estas actividades depende, en gran medida, de la organización interna del equipo, así como del grado de desarrollo del mismo y la prioridad de líneas de trabajo orientadas desde las Gerencias (Verbatim 14).

Cuando los grupos con mujeres se hacen «desde dentro» (esto es, con mujeres derivadas de las consultas) parecen tener un carácter más terapéutico y formativo y se hacen a iniciativa de personal de enfermería y/o trabajo social.

\section{Salud Escolar: «...también salimos fuera a los colegios"}

Otro ámbito de trabajo con grupos en instituciones, diferenciado de los anteriores, es el de la salud escolar. Aquí se incluyen tanto grupos de trabajo con maestros, pertenecientes a claustros de los colegios de la zona, como eventuales charlas a los propios niños de dichos colegios. En los grupos con maestros, normalmente a demanda de estos últimos y fuera del Centro de Salud, se enfatiza el apoyo técnico a las materias transversales (en concreto, la educación para la salud), basado en la transmisión de contenidos a los maestros para que ellos los elaboren 
con un carácter didáctico y, después, los trabajen con los niños. Los profesionales sanitarios que han participado en alguna actividad con maestros relatan que las principales dificultades radican alrededor de la falta de motivación de los mismos, así como en obstáculos de orden organizativo (Verbatim 15).

\section{Participación Comunitaria}

Bajo este amplio título aparecen en el discurso grupos de otro orden, más bien con el carácter de reuniones de coordinación, planificación, etc., que incluyen desde Consejos de Salud, Mesas de Salud, o trabajo conjunto con organizaciones e instituciones de la comunidad (asociaciones, ayuntamientos, Servicios Sociales, etc.) (Verbatim 16).

Las menciones a este tipo de actividades no son tan frecuentes como las anteriores (patologías crónicas, mujeres, etc.) y el debate sobre ellas pone de manifiesto una gran confusión acerca de qué es la participación comunitaria, llegando a constituir un «cajón de sastre» en el que pueden llegar a incluirse diversas actividades que tienen en común el que se realicen fuera del centro. De parte del colectivo de trabajo social surgen puntualizaciones que afinan la definición de «participación comunitaria», a menudo para resaltar que no es eso lo que se hace habitualmente (Verbatim 17).

\section{Formación de cuidadores}

De forma algo más minoritaria, se menciona que en algunos equipos se están llevando a cabo grupos con personas que cuidan o tienen a su cargo en el hogar enfermos graves, ancianos, «enfermos terminales», etc. Según se dice, estos grupos suelen estar compuestos por mujeres (que, en general, se encargan del cuidado de la salud familiar) $y$, desde un enfoque formativo y de apoyo, se procura asesorar y aconsejar sobre los cuidados específicos necesarios para la correc- ta atención de determinados enfermos, etc. Además, los profesionales que han tenido experiencia con estos grupos formativos apuntan que constituyen también un espacio de descanso y autoayuda para los cuidadores (Verbatim 18).

\section{DISCUSIÓN}

El uso de las técnicas cualitativas en la investigación en salud va siendo progresivamente aceptado en la comunidad científica sanitaria de nuestro país ${ }^{19-21}$ y su uso es creciente, entre otros en la evaluación de programas y actividades de salud ${ }^{22-25}$. Igualmente el grupo de discusión y las entrevistas abiertas están siendo cada vez más utilizados para conocer las perspectivas de los profesionales respecto a cuestiones organizativas ${ }^{26}$, para la investigación en atención primaria ${ }^{27,28}$ ó en determinados grupos de población ${ }^{29}$ (ver además las diferentes investigaciones cualitativas publicadas como Documentos Técnicos por la Consejería de Sanidad de la Comunidad de Madrid: http://www.documentacion.eps.comadrid.es). La complementariedad de métodos cuantitativos y cualitativos se va imponiendo, al menos en los discursos avanzados de la salud, en la perspectiva de obtener un conocimiento más comprehensivo y holístico de la problemática de la salud actual ${ }^{30-32}$.

En el caso del estudio que presentamos la principal limitación que encontramos, como en todos los estudios cualitativos, es la actualidad de sus resultados ya que el trabajo de campo fue realizado entre finales de 1995 y comienzos de 1996, aunque creemos que la opinión de los profesionales ha variado poco al respecto, si acaso se han polarizado todavía más las opiniones de cada colectivo implicado. Tampoco podemos generalizar sus resultados al referirse exclusivamente a los profesionales de la Comunidad de Madrid. El número de grupos y la combinatoria de los participantes satura suficientemente el universo del discurso ${ }^{33}$, aunque otras posibles 
combinaciones seguramente también hubieran aportado información relevante.

Desde la Administración la perspectiva de los profesionales generalmente ha sido poco tenida en cuenta a la hora de organizar las actividades que ellos mismos van a llevar a cabo, aunque cada vez más algunos de ellos participen en grupos de trabajo como expertos para revisar aspectos de política organizativa $^{34}$, implementar nuevos programas $^{35}$ o se hagan grupos focales para tener en cuenta su opinión en diferentes aspectos de la atención primaria ${ }^{36-38}$. Son pocos, pues, los estudios realizados en nuestro contexto para conocer las perspectivas de los profesionales de base, y todavía más escasos, ninguno que nosotros sepamos, sobre su opinión acerca del trabajo con grupos en atención primaria.

La consideración que los profesionales tienen de los grupos como algo marginal al sistema sanitario cobra pleno sentido cuando se analiza el desarrollo que ha tenido la atención primaria desde su puesta en marcha a mediados de los años 80 hasta finales de los noventa. Si bien en los planteamientos iniciales de la Reforma de la Atención Primaria se encontraban elementos (generalmente más en los discursos que en las prácticas) que hacían pensar en un fuerte desarrollo del trabajo preventivo en la comunidad, y por lo tanto de los grupos como dispositivos de intervención a potenciar por parte de equipos interdisciplinarios (los trabajadores sociales fueron la «novedad»), es a partir del Informe $\mathrm{Abril}^{5}$, y posteriormente con el desarrollo de la Cartera de Servicios, cuando se impone, a nivel del discurso y de las prácticas, el énfasis en la asistencia clínica individual y la «recuperación» de la hegemonía del «modelo médico» en la atención primaria ${ }^{39}$. Se opera así el pasaje de un modelo más ideológico y comunitario (de escaso desarrollo en sus aplicaciones prácticas) a un modelo más economicista y tecnocrático $^{40}$. De ahí que en los años inmediatamente posteriores al Informe Abril (1991) los argumentos del ahorro (de dinero, de tiempo, etc.) sean los dominantes, al tiempo que aumenta la preocupación por la demanda excesiva que «estallará» ya en el nuevo siglo (las sociedades científicas y numerosos profesionales han tomado como eje de sus reivindicaciones «los 10 minutos» por paciente). Los argumentos relacionados con el modelo de salud comunitaria, enarbolados principalmente por los profesionales de trabajo social (cuya contratación se estancó y cuya presencia ha ido quedando relegada a la «gestión de casos») y por algunos médicos y personal de enfermería, persisten sólo como «testimonio» de los primeros años de fervor comunitario.

Desde la propia Administración Sanitaria, y evidentemente esto les llega a los profesionales, se fomentan las prácticas asistenciales individuales que son las que mejor se prestan para cumplir las Normas Técnicas Mínimas necesarias para ser incluidas en la Cartera de Servicios, «reduciendo» las prácticas grupales a los Servicios 311 (Educación para la salud a grupos de pacientes crónicos) y 312 (Educación para la salud a otros pacientes). Es más, desde la evaluación de la Atención Primaria en estos diecisiete años ${ }^{41}$ no se echa en falta especialmente el trabajo grupal y en sus recomendaciones de líneas de futuro también brilla por su ausencia, aunque su carácter general (por ejemplo "flexibilizar la incorporación de servicios a la oferta», "seguir potenciando la implicación de los profesionales sanitarios», etc.) podría dar cabida a nuevos planteamientos de carácter grupal. Tampoco encontramos un entusiasmo mucho mayor cuando se plantean mejoras en Atención Primaria por parte de profesionales vinculados a Asociaciones Profesionales, Sindicatos, etc., en el marco de consenso con el Insalud $^{34}$. En este caso se hacen propuestas más explícitas algunas de ellas relacionadas con el trabajo grupal (Los profesionales de los Equipos de Atención Primaria deben implicarse en las actividades relacionadas con la Educación para la Salud y fomentar el autocuidado y la autonomía de los ciudadanos en el proceso de salud-enfermedad...»). 
Con todo, los profesionales «de base» se ven en el futuro (sin duda como expresión de lo que les gustaría que fuera) aumentando las actividades preventivas y más integrados en la comunidad ${ }^{37,42}$ e incluso algunos autores plantearon las posibilidades de hacer promoción de salud desde la Cartera de Servicios ${ }^{43}$. En otros estudios ${ }^{38,44}$ también los médicos señalan las dificultades para hacer educación para la salud en la atención primaria y centran sus dificultades en aspectos organizativos, burocráticos y de masificación en la consulta individual.

Respecto a los ámbitos de trabajo donde se hacen algunas actividades grupales coincidimos con diversos autores que señalan los grupos de enfermos crónicos ${ }^{9,45}$, de mujeres ${ }^{46,47}$, en parte en calidad de cuidadoras principales $^{48}$, y el ámbito educativo y comunitario como los ámbitos/grupos de población donde más frecuentes son las prácticas grupales en atención primaria.

En suma, la concepción de los grupos como un modo de «ahorro», al entenderlo como agregado de individuos, está más presente en aquellos colectivos que menos formación grupal han tenido durante su socialización universitaria (médicos/as, enfermeras/os y trabajadores sociales por este orden) y es la expresión máxima del enfoque de carácter biológico-individual que todavía domina nuestras instituciones académicas.

Los grupos, entendidos como tecnología de educación para la salud, pueden ser integrados en la práctica de la atención primaria como un recurso más en la atención «médica», pero en los profesionales encuentran mayores resistencias aquellas concepciones que entienden el trabajo con grupos como «otro modelo» de entender (y por lo tanto de atender) los problemas de salud-enfermedad, modelo éste que relativizaría el pa$\mathrm{pel} /$ poder de los profesionales para potenciar las capacidades de los individuos y el control sobre sus determinantes de salud (empowerment en terminología de la OMS) fomentando la participación, la comunicación interpersonal y la solidaridad entre las redes sociales ${ }^{49}$.

El enfoque de promoción de salud (que potenciaría esta segunda perspectiva grupal, incluyendo el trabajo en equipo) está lejos de ser incorporado en nuestro sistema sanitario y especialmente en la atención primaria de salud, ni por parte de la Administración sanitaria ni por los propios profesionales. En tiempos de globalización económica $\mathrm{y}$ de fragmentación social se produce un «retorno» del objetivismo médico ${ }^{50}$ y de las consiguientes modalidades asistenciales de carácter individualizante que «recortan» los problemas de salud al ámbito privado y a la relación médico/paciente, reforzando así la ruptura de los lazos sociales y la disminución de la participación social en salud. La representación social de los profesionales de atención primaria respecto a los grupos sería por lo tanto, a nivel microsocial, la expresión del discurso social dominante en lo referente a las relaciones sociales en general y a las relaciones profesionales/usuarios en particular.

A modo de resumen podemos señalar como conclusiones más relevantes que los discursos de los profesionales de atención primaria sobre los grupos se inscriben en un discurso más amplio sobre la atención primaria en salud en el cual los grupos se incluyen dentro de las actividades de educación para la salud, siendo ésta una actividad supeditada a la consulta individual. En segundo lugar las razones esgrimidas por los profesionales para hacer grupos incluyen argumentos del orden de la jerarquía (porque está en la Cartera de Servicios), de eficiencia (más rentables económicamente que la atención individual, descargan las consultas y ahorran tiempo), de efectividad (sirven para la prevención y los autocuidados) y de satisfacción personal (son gratificantes). Por último, los principales colectivos objeto del trabajo en grupo son, en orden decreciente, los pacientes crónicos (hipertensos y diabéticos), las mujeres (perimenopaúsicas, con 
malestar general, dolores, et.), los escolares y profesores, las asociaciones de la comunidad y los cuidadores de enfermos crónicos.

\section{AGRADECIMIENTOS}

A los profesionales de Atención Primaria de Madrid que han participado en los grupos de discusión y en las entrevistas sin cuyo discurso no podríamos hablar nosotros.

\section{BIBLIOGRAFÍA}

1. O'Shanahan JJ. Un Centro de Salud para el desarrollo. Tirajana 1976-1979. Rev Sanid Higiene Pública 1979; 53: 1595-1630.

2. Blanco P, Rodero J, Carro J, Vázquez J, López Alijo T y Fernández C. Experiencia sanitaria de Montánchez. Jano 1980; 389: 33-46.

3. Uriarte A y Ruiz-Giménez JL. Organización de un Centro de Salud a partir de la participación comunitaria. Atención Primaria. 1993; 11: 253-7.

4. Duro JC. Psicología y Salud Comunitaria durante la Transición Democrática. Clínica y Salud 2001; 12: $1-175$.

5. Instituto Nacional de la Salud. Comisión de Análisis y Evaluación del Sistema Nacional de Salud. Informe y Recomendaciones. Madrid: Instituto Nacional de la Salud, 1991.

6. Insalud. Cartera de Servicios en Atención Primaria. Definiciones, Criterios de Acreditación, Indicadores de Cobertura y Normas Técnicas Mínimas. Actualización en 2000, 4. ${ }^{\mathrm{a}}$ edición. Madrid: Instituto Nacional de la Salud; 1995.

7. Lucas A y González Tapias JL. Los Centros de Promoción de la Salud: una experiencia positiva en la Atención Primaria de Salud. Revista Internacional de Sociología 1982; 40: 137-152.

8. Ruiz-Giménez JL. Trabajo comunitario y participación en Atención Primaria. Líneas de trabajo en el Área 1 del Insalud de Madrid. Boletín de Ayuda Mutua y Salud 1995; 1: 81-8.

9. Zamarra MP. Educación para la Salud a grupos en Atención Primaria. Una experiencia satisfactoria sobre autocuidados para el asma en Pediatría. Centro de Salud 1996; 4: 658-63.

10. Carrasco E y Puig de la Bellacasa J. Grupos de Educación para la Salud en un programa de Salud
Escolar. En: Modelos grupales en Psicoterapia: aspectos teóricos y técnicos. Madrid: SEGPA; 1990. p. 131-7.

11. Ibáñez J. Más allá de la sociología. El grupo de discusión: técnica y crítica. 2. ${ }^{a}$ edición corregida Madrid: Siglo XXI; 1986.

12. Ibáñez J. Autopercepción intelectual de un proceso histórico. Autobiografía (Los años de aprendizaje de Jesús Ibáñez). Anthropos 1990; 47: 9-26.

13. Delgado JM, Gutiérrez J. Métodos y técnicas cualitativas de investigación en ciencias sociales. Madrid: Síntesis; 1994.

14. Vallés MS. Técnicas cualitativas de investigación social. Reflexión metodológica y práctica profesional. Madrid: Síntesis; 1997.

15. Fernández de Sanmamed MJ. Introducción a la investigación cualitativa. Aten Primaria. 1995; 2: $23-8$.

16. Ortí A. La apertura y el enfoque cualitativo o estructural: La entrevista abierta semidirectiva y la discusión de grupo. En: Alvira F, García Ferrando M, Ibáñez J, editores. El análisis de la realidad social. Madrid: Alianza Universidad; 1989; p. $153-185$.

17. Alonso LE. Sujeto y discurso: el lugar de la entrevista abierta en las prácticas de la sociología cualitativa. En: Delgado JM, Gutiérrez J, editores. Métodos y técnicas cualitativas de investigación en Ciencias Sociales. Madrid: Síntesis; 1994; p. 225-420.

18. Varios Autores. Investigación-Acción-Participativa. Documentación Social 1993; 92.

19. Fernández de Sanmamed MJ. Métodos y técnicas cualitativas en la investigación en atención primaria. Aten Primaria 1999; 23: 453-4.

20. Martín Zurro A. Nuevas perspectivas en la evaluación de la atención primaria: las metodologías cualitativas. Aten Primaria. 2000; 25: 605.

21. García Calvente MM y Mateo Rodríguez I. El grupo focal como técnica de investigación en salud: diseño y puesta en práctica. Aten Primaria 2000; 25: 181-6.

22. Fernández Sierra J; Santos MA. Evaluación cualitativa de programas de educación para la salud. Una experiencia hospitalaria. Málaga: Aljibe; 1992.

23. Santos MA, Prados JD. Evaluación externa de la formación de médicos residentes: el arte de mejorar a través del conocimiento. Barcelona: Socie- 
dad Española de Medicina Familiar y Comunitaria; 1996.

24. Duro JC y Molina MM. La Consulta Joven en los Centros de Salud de Fuenlabrada (Madrid): una evaluación cualitativa. Medifam 1998; 8: 37-49.

25. Sánchez-Candamio M. Evaluación de programas de salud desde la perspectiva de la metodología cualitativa. Atención Primaria. 1999; 24 487-491.

26. Pérez Andrés C. Aceptación del Contrato Programa por el personal sanitario de la Atención Primaria de Madrid: un estudio cualitativo. Rev San Hig Pública 1995; 69: 79-88.

27. Buzzaqui A, Uris J. El grupo de discusión. Una herramienta para la investigación en Atención Primaria. Aten Primaria 1997; 4: 421-33.

28. Ribot C, Fernández Tenllado MA y García de León D. Investigación cualitativa en Atención Primaria. Una experiencia con entrevistas abiertas. Aten Primaria. 2000; 25: 343-8.

29. Álvarez MI, Benito R, Cristóbal AM, Duro JC Germain J y Valgoma A. La salud en la vejez: un discurso optimista. Revista de Gerontología 1997; 7: 177-82.

30. Health Education Quaterly. Integrating quantitative and qualitative methods. Health Education Quaterly. John Wiley \& Sons, Inc.; 1992; 19 (1).

31. Consejería de Salud. Informe sobre la Salud y la Mujer en la Comunidad de Madrid. Madrid: Consejería de Salud; 1996. Documentos técnicos de salud pública, núm. 32.

32. Baum F. Investigación en salud pública: el debate sobre las metodologías cuantitativas y cualitativas. Revisioes en Salud Pública. 1997; 5: 175-193

33. Ibáñez J. ¿Cómo se hace una investigación mediante grupos de discusión? En: García Ferrando M, Ibáñez J, Alvira F, editores. El análisis de la realidad social. Madrid: Alianza Editorial; 1990 p. 489-501.

34. Subdirección General de Atención Primaria Insalud. Los Equipos de Atención Primaria. Propuestas de mejora. Documento de consenso. Madrid: Instituto Nacional de la Salud; 2001.

35. Insalud. Desarrollo de planes de cuidados en la cartera de servicios de Atención Primaria. Madrid: Instituto Nacional de la Salud; 2001.

36. Agra Y, Arévalo T, Campanero MP, González F y Sánchez A. Estrategias para la mejora de los servicios de admisión de Atención Primaria: utilidad de los grupos de discusión. Medifam 1996; 6 : 162-7.

37. Sarmiento Gallego M, Martínez Ros MT, Sánchez Sánchez F, Sánchez Esteban JM, Messeguer Zaragoza A y Jiménez Bello JJ. ¿Cómo vemos los sanitarios el previsible futuro de nuestro trabajo en los centros de salud? Aten Primaria 1997; 20: $311-4$.

38. Turabián JL y Pérez-Franco B. Los problemas y las soluciones de los médicos de familia en su trabajo: un estudio de grupos focales con tutores de Medicina de Familia y Comunitaria. Medifam 1998; 8: 369-75.

39. Menéndez E. Jornadas de Antropología y Salud Pública. 1. ${ }^{\mathrm{a}}$ Jornadas de Promoción y Educación para la Salud. Madrid: Centro Universitario de Salud Pública; 1993; Atención Primaria: las propuestas y las posibilidades. p. 1-14.

40. Duro JC. Discursos y prácticas profesionales de psicología social en salud comunitaria (1970-1995) [Tesis doctoral]. Departamento de Sociología y Antropología Social. Facultad de Ciencias Económicas y Empresariales. Madrid: Universidad Autónoma de Madrid; 1999.

41. Insalud. Atención Primaria en el Insalud: Diecisiete años de experiencia. Madrid: Instituto Nacional de la Salud; 2002.

42. Rodríguez R, Márquez S y López S. La perspectiva profesional en la reforma de la atención primaria de salud: una aproximación cualitativa. Gac Sanit 1995; 9: 237-43.

43. Turabián JL, Pérez-Franco B, Díaz L, Colino R y Menéndez-Cabeza MV. Desde la cartera de servicios a la promoción de la salud. Centro de Salud 1994; 2: 535-9.

44. Díaz Grávalos GJ. Factores relacionados con la práctica de Educación sanitaria por los médicos de atención primaria de Galicia. Rev Esp Salud Pública 2001; 75: 529-40.

45. Lozano del Hoyo ML y al. Diabetes tipo II no insulinodependientes. Educación grupal. Revista de Enfermería 1997; 19: 75-8.

46. De Argila N, Alba R. Experiencia educativa con mujeres con pluripatología en el Centro de Salud Doctor Cirajas. Barbacana. Boletín de AP del Area 4.Insalud-Madrid 1997; 24: 19-21.

Rev Esp Salud Pública 2002, Vol. 76, N. ${ }^{\circ} 5$ 
EL DISCURSO DE LOS PROFESIONALES DE ATENCIÓN PRIMARIA DE LA COMUNIDAD...

47. Conde F. Las mujeres y el movimiento de ayuda mutua. Boletín de Ayuda Mutua y Salud 1997; 3: 34-53.

48. Martín C, Medina M e Irazábal E. ¿Quién cuida al cuidador? Barbacana. Boletín de AP del Area 4.Insalud-Madrid 1996; 19: 94-95.
49. Ministerio de Sanidad y Consumo/OMS. Promoción de la salud. Glosario. Madrid: Ministerio de Sanidad y Consumo/OMS; 1998.

50. Castel R. La gestión de los riesgos. De la anti-psiquiatría al post-análisis. Barcelona: Anagrama; 1984. 